\title{
The First Report of Fusarium solani Causing Wilting in Cnidium officinale in Korea
}

*Corresponding author

Tel: +82-54-630-5642

Fax: +82-54-630-5678

E-mail: crjung92@korea.kr
Received May 19, 2021

Revised June 26, 2021

Accepted June 27, 2021

\author{
Kyeungmin Han ${ }^{1}$, Hyoshin Lee ${ }^{2}$, Yun Mi Park ${ }^{3}$, Kwon Seok Jeon ${ }^{4}$, Wonsu Choen ${ }^{5}$, \\ and Chung Ryul Jung ${ }^{4 *}$
}

${ }^{1}$ Department of Forest Plant Industry, Baekdudaegan National Arboretum, Bonghwa 36209, Korea.

${ }^{2}$ Forest Bioinformatics Division, Forest Bioresources Department, National Institute of Forest Science, Suwon 16631, Korea

${ }^{3}$ Special Forest Resources Division, Forest Bioresources Department, National Institute of Forest Science, Suwon 16631, Korea

${ }^{4}$ Forest Medicinal Resources Research Center, National Institute of Forest Science, Yeongju 360040, Korea

${ }^{5}$ Fungi Research Team, Microbial Research Department, Nakdonggang National Institute of Biological

Resources, Sangju 37242, Korea

Cnidium officinale is a perennial herb belonging to the family Umbelliferae. Its origin is China and is also distributed in Korea and Japan. In 2017, a phenomenon of browning and wilting of leaves was discovered in the cultivation field of the National Institute of Forest Science, Suwon, Korea. The pathogens isolated from plants were consistent with Fusarium solani as a result of morphological examination and molecular phylogenetic analysis. This is the first record of $F$. solani on $C$. officinale in Korea.

Keywords: Cnidium officinale, Fungal disease, Fusarium solani, Fusarium wilt

Cnidium officinale Makino is a perennial crop that belongs to the Apiaceae family and it is used as a natural medicine in Asia. C. officinale has been reported to possess anticancer, antioxidant, and platelet aggregation inhibition properties. Therefore, health-related research on C. officinale is conducted in food processing, pharmaceutical, and cosmetic industries. In Korea in 2017, approximately 1,297 tons of $C$. officinale were produced, making this plant the fourth most abundate herbal medicines (Ministry of Agriculture, Food and Rural Affairs, 2018). However, plant diseases reduce its production and affect the growth of the roots that are used as medicine. Erwinia rhapontici, Colletotrichum gloeosporioides, and Fusarium sp. have been reported as pathogens of $C$.

Research in Plant Disease

pISSN 1598-2262, elSSN 2233-9191

www.online-rpd.org officinale in Korea. And so far, the pathogen causing leaf wilt symptoms in C. officinale has been identified as Fusarium sp.

In 2017, we observed that the leaves of $C$. officinale turned brown and wilted in the cultivation field of the Forest Bioresources Department, National Institute of Forest Science, Suwon, Korea. The symptoms initially appeared as small, brown lesions on the edges of the leaves (Fig. 1A). After 2 or 3 weeks, the entire plant had wilted and dried up (Fig. 1B). To isolate potential pathogens from the infected leaves and roots, small sections $\left(5 \mathrm{~mm}^{2}\right)$ were excised from the lesions. These sections were surface-disinfested with $1 \% \mathrm{NaOCl}$ for $1 \mathrm{~min}$ and were then rinsed thoroughly with sterile-distilled water. These fungi were then transferred to water agar and potato dextrose agar (PDA) plates and were incubated at $25^{\circ} \mathrm{C}$. The isolated strain developed white or brownish submerged hyphae (Fig. 1C) in cloud form and appeared to contain macroconidia and microconidia (Fig. 1D). The mac- 


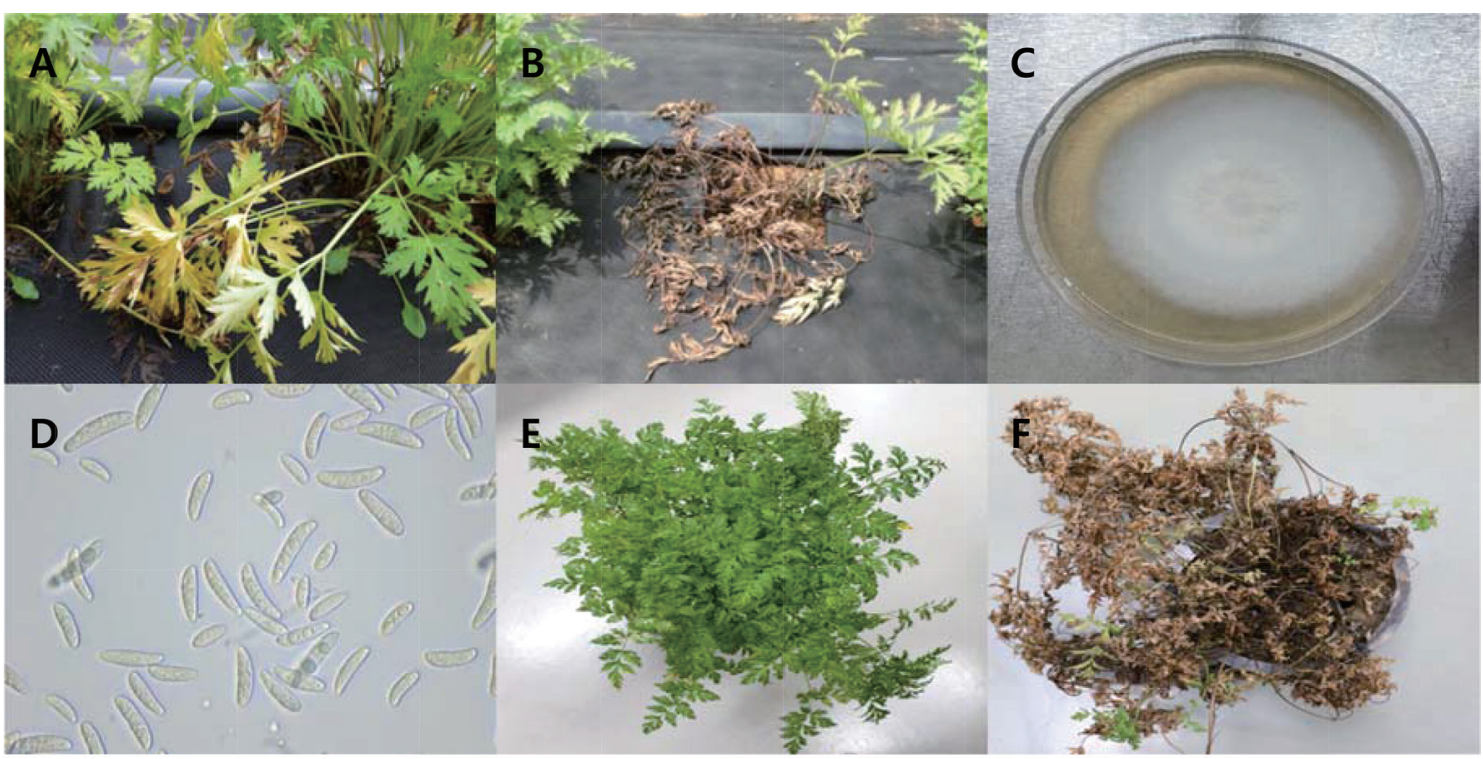

Fig. 1. Symptoms of Fusarium wilt caused by Fusarium solani and morphological characteristics of $F$. solani. (A) Occurrence of Fusarium wilt in a farm in April 2017. Wilted plants among the healthy (B), colony morphology on potato dextrose agar after 10 days of incubation (C), macroconidia and microconidia (D) and pathogenicity test of the isolated pathogen on Cnidium officinale $(E, F)$.

roconidia were relatively wide and straight, had 3 to 6 septa, and measured $21-65 \times 3-5 \mu \mathrm{m}$. The microconidia were either ovoid, ellipsoidal, or fusiform, with 0,1 , or occasionally 2 septa, and measured 8-18 $\times 2-5 \mu \mathrm{m}$. These morphological characteristics match those of $F$. solani. Pathogenicity tests were performed by spraying a conidvial suspension $\left(10^{5}\right.$ conidia/ $\mathrm{ml}$ or sterile water for the control) into the healthy plant pots. After inoculation, the plants were kept in a growth chamber at $25^{\circ} \mathrm{C}$ with a relative humidity of $80-90 \%$. After 5 days, yellow discoloration was observed on plant leaves, and 7 days later, their leaves had turned brown and wilted, which is similar to what is observed on naturally infected leaves (Fig. $1 \mathrm{E}, \mathrm{F})$. The pathogens were re-isolated from the symptomatic tissues and their colonies were cultured on PDA once more. Molecular analysis was performed to support the morphological characteristics identified earlier. Fungal isolates

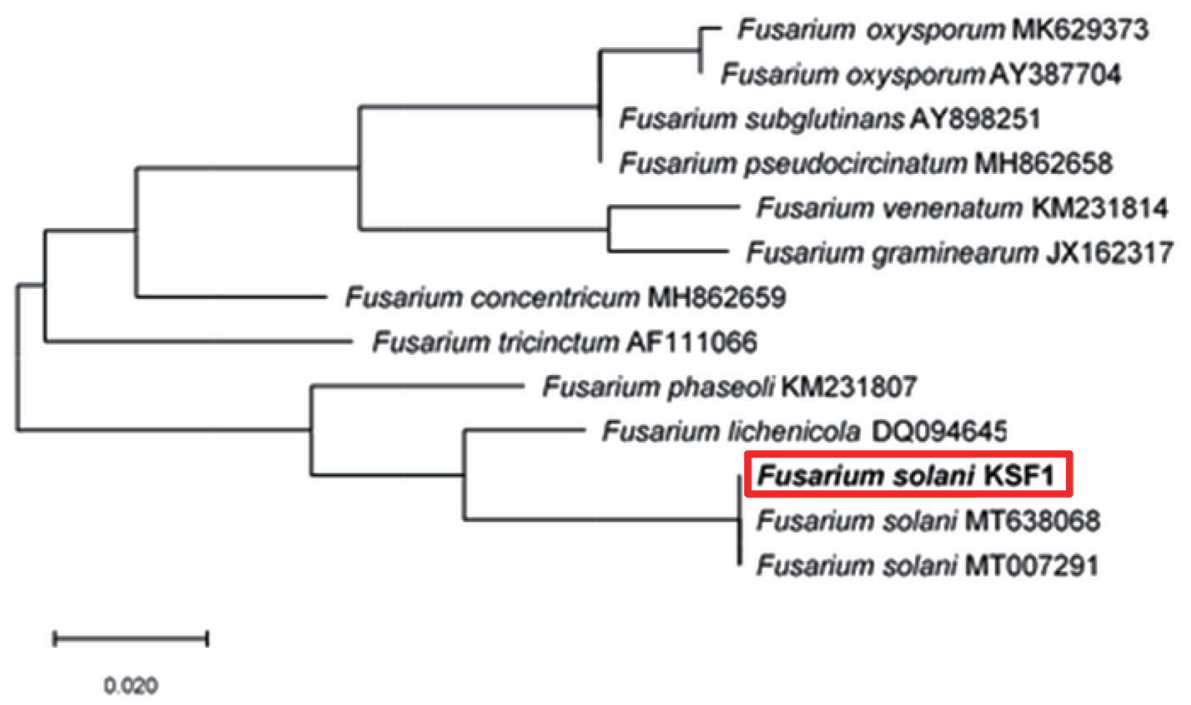

Fig. 2. Phylogenetic relationship between Fusarium solani and other Fusarium species, constructed using the maximum likelihood method and Tamura-Nei model (Tamura and Nei, 1993), based on the combined internal transcribed spacer region gene sequences. 
were characterized by sequencing their internal transcribed spacer (ITS) rDNA region using ITS4/ITS5 primers (Gardes and Bruns, 1993; White et al., 1990) on SolGent Co., Ltd. (Daejeon, Korea). The sequences of isolate (accession no. MK934409) matched F. solani (accession nos. MT638068, MT007291) sequences in GeneBank with a similarity score of $99 \%$ (Fig. 2). For phylogenetic analysis, the phylogenetic tree for the nucleotide sequence in the ITS region was prepared by the maximum likelihood method using MEGA X (Fig. 2) (Kumar et al., 2018), and the genetic distance of the nucleotide sequence was calculated using the Tamura-Nei model, and bootstrap analysis was performed with 1,000 iterations. The $F$. solani KFS1 strain used in this study was deposited with the Korean Agricultural Culture Collection (KACC) under the accession number KACC49599. The morphological characteristics, pathogenicity, and molecular data of the fungal isolates corresponded with those of $F$. solani. To our knowledge, this is the first report of $F$. solani as a pathogen of $C$. officinale in Korea.

\section{Conflicts of Interest}

No potential conflict of interest relevant to this article was reported.

\section{Acknowledgments}

This work was supported by the grants from 'Forest Science and Technology Development' (Project No. FE0702-2018-02) funded by the Korea Forest Service, Republic of Korea.

\section{References}

Gardes, M. and Bruns, T. D. 1993. ITS primers with enhanced specificity for basidiomycetes: application to the identification of mycorrhizae and rusts. Mol. Ecol. 2: 113-118.

Kumar, S., Stecher, G., Li, M., Knyaz, C. and Tamura, K. 2018. MEGA $X$ : Molecular Evolutionary Genetics Analysis across computing platforms. Mol. Biol. Evol. 35: 1547-1549.

Ministry of Agriculture, Food and Rural Affairs. 2018. An Actual Output of Crop for a Special Purpose in 2017. Ministry of Agriculture, Food and Rural Affairs, Sejong, Korea. 21 pp. (in Korean)

Tamura, K. and Nei, M. 1993. Estimation of the number of nucleotide substitutions in the control region of mitochondrial DNA in humans and chimpanzees. Mol. Biol. Evol. 10: 512-526.

White, T. J., Bruns, T., Lee, S. and Taylor, J. 1990. Amplification and direct sequencing of fungal ribosomal RNA genes for phylogenetics. In: PCR Protocols: A Guide to Methods and Applications, eds. by M. A. Innis, D. H. Gelfand, J. J. Sninsky and T. J. White, pp. 315-322. Academic Press, San Diego, CA, USA. 